\title{
On-board Evaluation Results of Active Phased Array Antenna for WINDS Satellite
}

\author{
By Masanobu YAJIMA ${ }^{1)}$, Takumi HASEGAWA ${ }^{2)}$, Tomonori KURODA ${ }^{3)}$ and Masaaki SHIMADA ${ }^{1)}$ \\ ${ }^{1)}$ Telecommunications and Data Handling Group, JAXA, Tsukuba, Japan \\ ${ }^{2)}$ Network Control Division, Space Engineering Development Co., Ltd, Tsukuba, Japan \\ ${ }^{3)}$ Satellite Applications and Promotion Center, JAXA, Tsukuba, Japan
}

(Received June 12th, 2009)

Phased Array Antennas (PAAs) have attracted attention for use as hopping spot beam antennas. In fact, a Ka band APAA was installed on the KIZUNA (WINDS). It was launched by an H-IIA Launch Vehicle in February 2008. This paper describes the WINDS APAA configuration, in addition to development results and on-board evaluation results of the APAA flight model.

Key Words: APAA, WINDS, Hopping Spot Beam Antennas, $622 \mathrm{Mbps}$

\section{Introduction}

Phased Array Antennas (PAAs) have attracted attention for use as hopping spot beam antennas onboard satellites, that may be electronically controlled. In fact, a Ka band APAA ${ }^{1)}$ was installed on the KIZUNA (Wideband Internetworking engineering test and Demonstration Satellite: WINDS) ${ }^{2)}$ which was developed by JAXA and the National Institute of Information and Communications Technology (NICT). Actually, WINDS was developed to construct a high-speed satellite communication system and demonstrate the advanced technology that is necessary for the creation of a satellite communications network. It was launched by an H-IIA Launch Vehicle in February 2008.

The APAA comprises a transmitting (TX) array antenna and a receiving (RX) array antenna; each antenna has two hopping spot beams. Each beam's direction can be controlled independently, flexibly and rapidly. These beams can also perform satellite switched time division multiple access (SS-TDMA) communication functions. The hopping spot beam function and SS-TDMA communication systems will be used for broadband communication experiments covering the Asia-Pacific region.

This paper describes the WINDS APAA configuration, in addition to the development results and on-board evaluation results of the APAA flight model.

\section{APAA Configuration}

Figure 1 portrays the APAA configuration. This is composed of a TX array antenna, an RX array antenna, a beam steering controller (BSC), DC/DC converters, heaters, a heater control unit (HCU), and power distribution units (PDUs). The transmitting and receiving antennas are mounted on the same surface of the APAA Earth panel. All components except the antennas are mounted in the antenna structure unit, which comprises aluminum honeycomb panels having heat pipes and thermal radiation plates. The APAA dimensions are $1,420 \mathrm{~mm} \times 920 \mathrm{~mm} \times 1,500 \mathrm{~mm}$; its mass is $183 \mathrm{~kg}$. The APAA has a self-controlled thermal dissipation function to maintain a specified temperature range.

Its required performance is presented in Table 1. The TX array antenna is a transmitting antenna for the downlink;

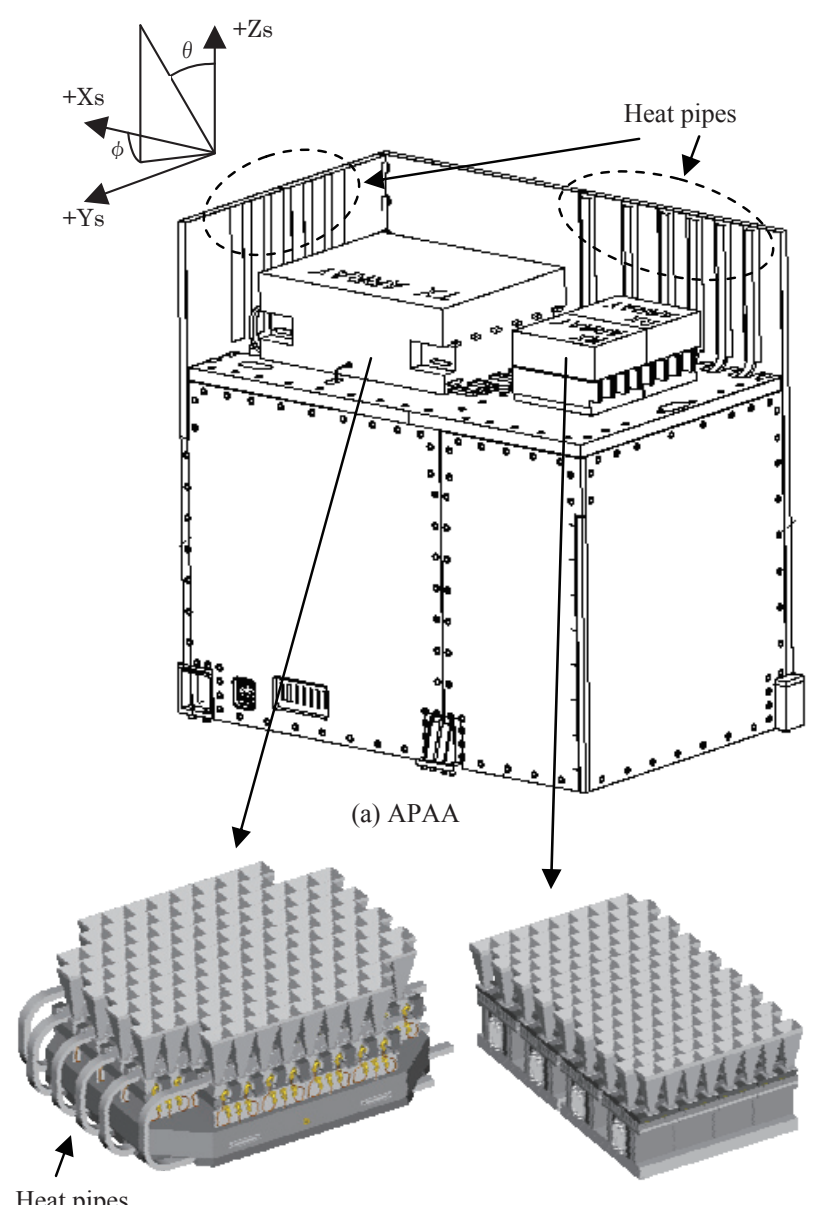

Heat pipes

(b) TX array antenna

(c) RX array antenna

Fig. 1. APAA configuration.

the $\mathrm{RX}$ array antenna is a receiving antenna for the uplink. The Ka-band frequency bandwidth is $1.1 \mathrm{GHz}$ for TX and RX. Each antenna provides two multiple beams for direction within an ellipse: 8 degrees for azimuth and 7 degrees for elevation. Both antennas are designed to provide the specified gain with a minimum number of antenna elements and are designed to avoid grating lobes on the Earth's surface. Considering these conditions, we used a 128-element array antenna with a triangular lattice. Each element spacing is 


\begin{tabular}{|c|c|c|c|}
\hline Item & Unit & Performance & Remarks \\
\hline Antenna type & - & $\begin{array}{c}\text { Direct radiating } \\
\text { phased array antenna }\end{array}$ & \\
\hline Aperture size & $\mathrm{mm}$ & $\begin{array}{l}649 \times 539(\mathrm{TX}) \\
287 \times 468(\mathrm{RX})\end{array}$ & \\
\hline Frequency band & $\mathrm{GHz}$ & $\begin{array}{l}18(\mathrm{TX}) \\
28(\mathrm{RX})\end{array}$ & \\
\hline $\begin{array}{l}\text { Frequency } \\
\text { bandwidth }\end{array}$ & $\mathrm{GHz}$ & 1.1 & \\
\hline $\begin{array}{l}\text { Number of } \\
\text { radiation } \\
\text { elements }\end{array}$ & - & 128 & \\
\hline Polarization & - & Linear & \\
\hline Field of view: FOV & $\operatorname{deg}$ & $\begin{array}{l}\text { Azimuth: } 8 \\
\text { Elevation: } 7\end{array}$ & Ellipse \\
\hline Number of beams & - & 2 & \\
\hline $\begin{array}{l}\text { Equivalent } \\
\text { isotropic } \\
\text { radiated power: } \\
\text { EIRP }\end{array}$ & $\mathrm{dBW}$ & $\begin{array}{l}\geq 54.6 / 1 \text { beam } \\
\geq 52.1 / 2 \text { beams }\end{array}$ & $\begin{array}{l}\text { Beam center } \\
\text { in FOV }\end{array}$ \\
\hline $\begin{array}{l}\text { Antenna gain to } \\
\text { noise temperature } \\
\text { ratio: } \mathrm{G} / \mathrm{T}\end{array}$ & $\mathrm{dB} / \mathrm{K}$ & $\geq 7.1$ & $\begin{array}{l}\text { Beam center } \\
\text { in FOV }\end{array}$ \\
\hline Directive gain & $\mathrm{dBi}$ & $\geq 37.8$ & at $\theta=0$ \\
\hline $\begin{array}{l}\text { Phase shifter bit } \\
\text { number }\end{array}$ & bit & 5 & $\begin{array}{l}\text { Digital phase } \\
\text { shifter }\end{array}$ \\
\hline Beam hopping time & $\mu \mathrm{s}$ & $\leq 10$ & at SS-TDMA \\
\hline
\end{tabular}

$2.7 \lambda$, where $\lambda$ is the wavelength. A pyramidal horn antenna was adopted as the antenna element because of requirements for a broad bandwidth and high efficiency. The amplitude distributions are uniform in order to achieve the required antenna gain.

As shown in the detailed block diagram of the APAA in
Figure 2, the TX array antenna is composed of 128 horn antennas, 128 high power amplifiers (HPAs), and a beam forming network (BFN) with 256 digital phase shifters (PSs) for forming two beams independently. Each RF signal into the beam input port is amplified and distributed to 128 way ports independently. The distributed signals are shifted to the desired phase and combined with both beams. The combined signals are amplified by the HPAs, having an output power of $28 \mathrm{dBm}$, and are radiated by the horn antennas.

The RX array antenna is also composed of 128 horn antennas, 128 low noise amplifiers (LNAs), and a BFN with 256 digital PSs for forming two beams independently. Each LNA is used as a common amplifier for two beams, and two sets of PSs and a combiner form the two beams.

The BSC independently controls the phase of all PSs in the transmitting and receiving antennas at $2 \mathrm{~ms}$ intervals, so each antenna radiates two independent scanning beams in accordance with SS-TDMA operations. 8 scanning spot beam areas for each beam can be selected in SS-TDMA. Also, the BSC controls the attenuators in the BFNs to compensate for gain variations due to temperature.

The DC/DC converters convert the 50 -Volt bus voltage to each secondary low voltage. The conversion efficiency is exceeded by up to $80 \%$.

The HCU will ensure that the heaters are functioning within the operating temperature. The heaters are composed of a replacement heater controlled by ON/OFF commands, and a thermal control heater controlled automatically by setting the temperature. The heat radiation areas are optimized by adopting a heat pipe with a three-dimensional heat sink system. This design makes it possible to downsize the APAA.

APAA

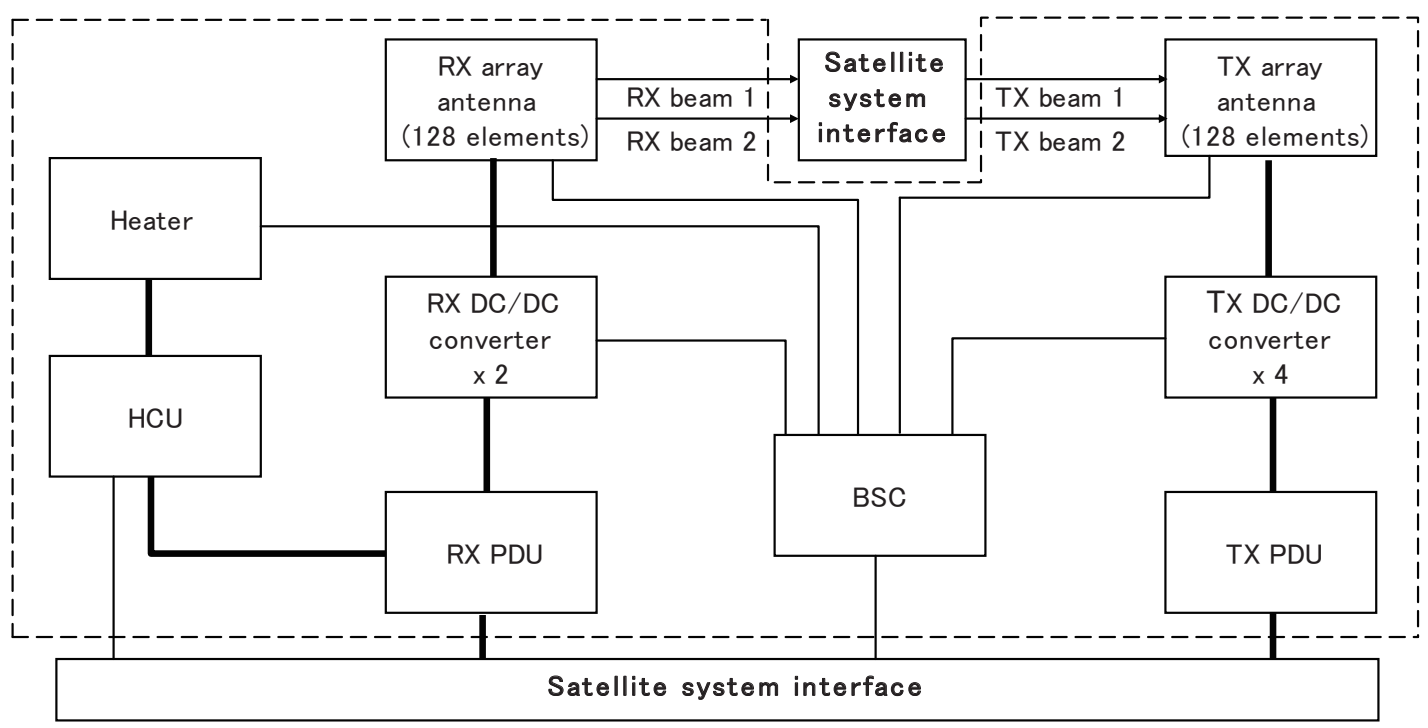

$\mathrm{HCU}$ : Heater control unit

PDU : Power distribution unit

BSC : Beam steering controller

Fig. 2. APAA block diagram. 


\section{Development Results}

The measured radiation patterns of the APAA flight model are presented in Figure 3. Good correspondence is shown between measured patterns and calculated ones, enabling confirmation of the performance of the two beams. Actually, EIRP is $56.0 \mathrm{dBW}$ ( 1 beam), and G/T is $8.5 \mathrm{~dB} / \mathrm{K}$. We also confirmed the beam hopping capability for $2 \mathrm{~ms}$ TDMA communication systems and an antenna gain control performance for the gain variation compensation attributable to temperature change.

Figure 4 shows the APAA frequency characteristics. The EIRP and RX array antenna gain values in the case of $\theta=8$ degrees are lower than those in the case of $\theta=0$ degrees because of the element factor in the APAA. The RX array antenna gain values include the antenna gain and the amplifier gain. These values also satisfy the APAA performance requirements.

\section{On-board Evaluation Results}

We tested and evaluated an APAA flight model on the WINDS satellite by using some earth stations. We also performed broadband communication experiments with this model. The items evaluated are shown below.

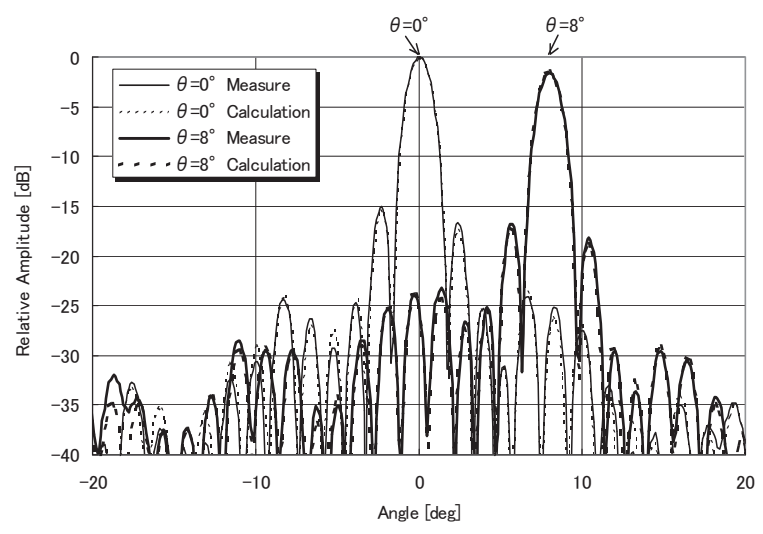

(a) TX array antenna

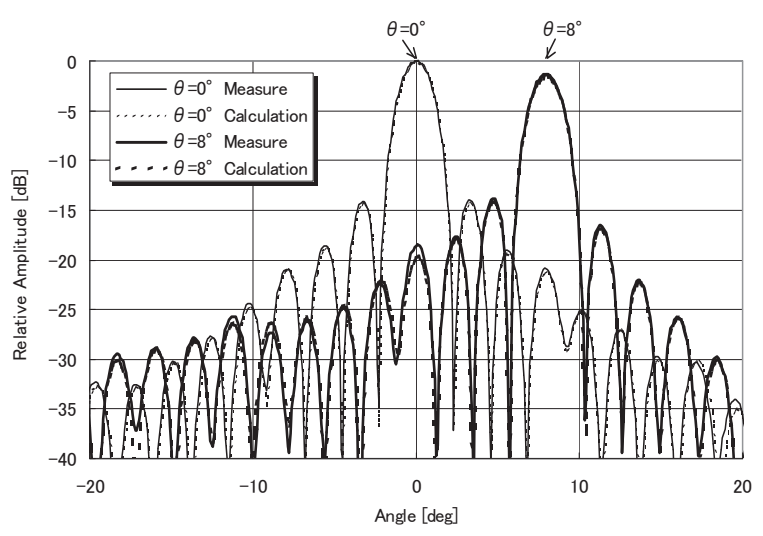

(b) RX array antenna

Fig. 3. Radiation patterns of antennas.

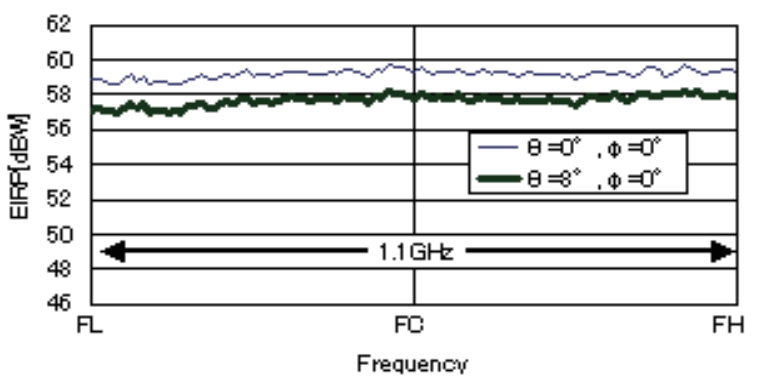

(a) TX array antenna

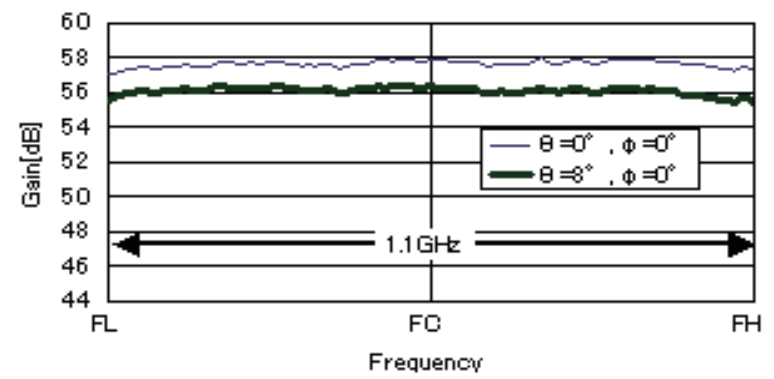

(b) RX array antenna

Fig. 4. Frequency characteristics.

(a) APAA basic functions and specifications confirmation.

(b) $622 \mathrm{Mbps}$ bent-pipe communication experiment in a continuous wave operation mode.

(c) 24/ 1.5 Mbps regenerative switching communication experiment in an SS-TDMA operation mode.

(d) Antenna radiation patterns confirmation (only main-lobe).

(e) APAA health check confirmation using a Rotating element Electric field Vector (REV) method ${ }^{3)}$.

\subsection{APAA basic functions and specifications confirmation}

The turn on/off test results of all components except redundancy heaters were normal. Storage data in the BSC after turn on were transmitted normally, and the APAA control and monitor functions were performed. The temperature of all components was within the expected temperature range, and the heater thermal control function was normal.

\subsection{Mbps bent-pipe communication experiment}

Figure 5 shows the bent-pipe communication experiment configuration. We successfully achieved a high data rate communication at a speed of $622 \mathrm{Mbps}$ using the APAA between the Large Aperture Terminal ( $5 \mathrm{~m}$ diameter antenna) in the NICT Kashima Space Research Center and a super high data rate earth station ( $2.4 \mathrm{~m}$ diameter antenna) set on a car in Kushiro. It is the fastest speed in the world using an on-board APAA.

We also calculated the APAA EIRP and G/T values by using receiving signals and noise pedestals of WINDS signal relay components at Kushiro. It is difficult to derive the exact EIRP and G/T values because of changes in weather conditions, shifts in antenna pointing direction, and so on, but their values are above the specification values, as shown in 


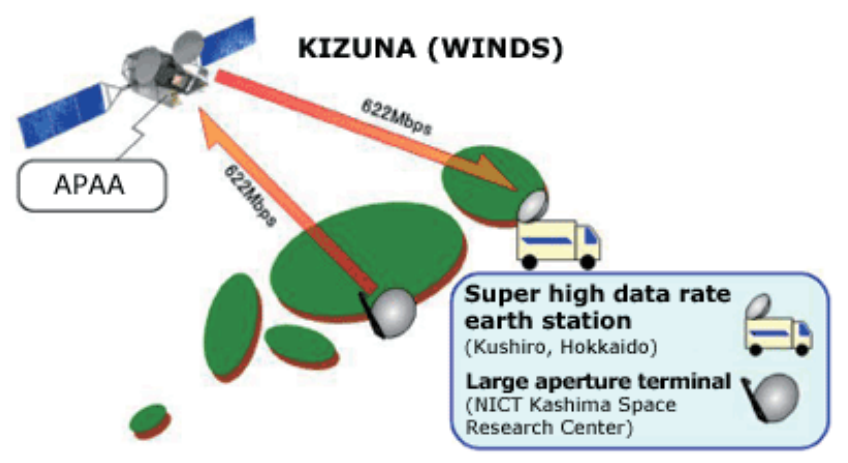

Fig. 5. 622 Mbps bent-pipe communication experiment configuration.

table 1. The APAA beam control function and the Ka band signal receiving and transmitting function were also normal.

\subsection{4/ 1.5 Mbps regenerative switching communication experiment}

The experiment configuration is the same as the configuration of figure 5 except for the satellite data relay function. In this experiment, the satellite regenerative switching function was used because the APAA operation mode was SS-TDMA. We successfully achieved a $24 \mathrm{Mbps}$ communication from the Kashima station to the Kushiro station by using APAA TX/RX beam \#1, and a $1.5 \mathrm{Mbps}$ communication from Kushiro to Kashima by using TX/RX beam \#2.

Next, we performed a beam hopping experiment by replacing the transmission direction from Kashima to Kushiro with the direction from Kushiro to Kashima in 2 ms intervals alternately. This experiment was also successful.

\subsection{Antenna radiation patterns confirmation}

We acquired and analyzed the TX/RX antenna beam radiation patterns by scanning their beams around the earth station. Figures 6 and 7 show the results of these radiation patterns. Based on figures 6 and 7, the results were good, considering that the measured resolution of the $\mathrm{CW}$ signal level was $0.5 \mathrm{~dB}$.

\subsection{APAA health check confirmation}

It is a feature of the APAA that many antenna elements are arranged on the antenna aperture, and active devices such as amplifiers and PSs are connected to its elements. Therefore, it is difficult to monitor the output of each amplifier directly, so the REV method is effective in performing a health check of each element of the APAA.

The REV method is explained as follows: at first, the phase setting of one element is changed by 1 bit by means of a digital PS in a transmitting APAA, and the level of the RF signal received by an antenna on the opposite side is monitored. Next, the condition of each element is assessed; if the RF signal level of one element forms a cosine curve, the element is normal; otherwise it is not normal.

The WINDS APAA can perform the REV method on orbit by monitoring the RF signal level received at an earth station. It has an automatic phase setting mode function that can be set by command from the station. In this mode, the phase setting of each element is automatically changed by 1 bit, that is,

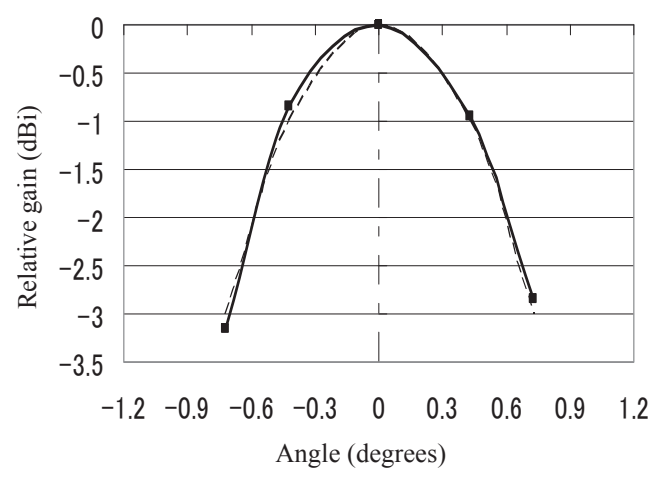

(a) East-west direction (Beam \#1)

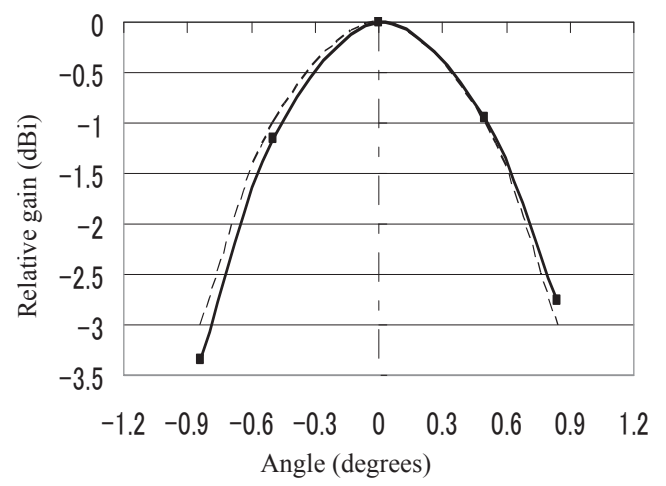

(b) North-south direction (Beam \#1)

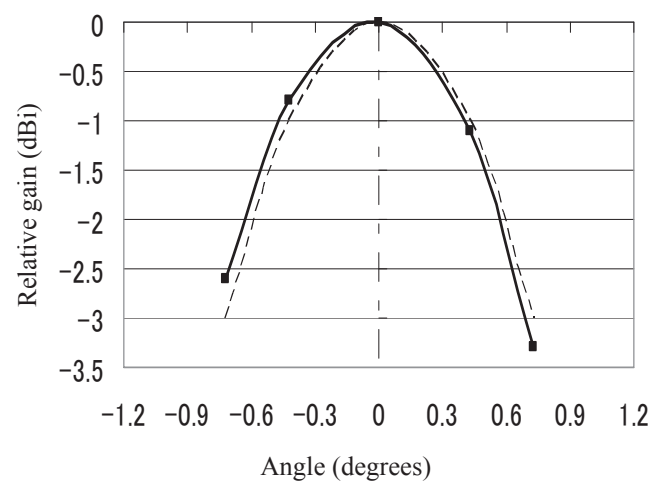

(c) East-west direction (Beam \#2)

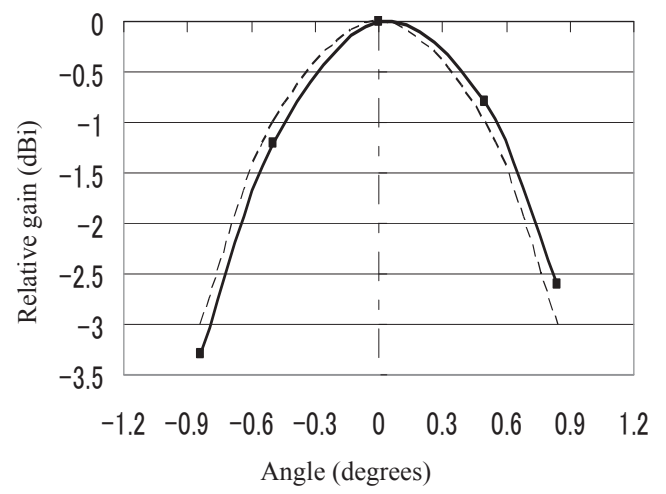

(d) North-south direction (Beam \#2)

Fig. 6. TX Antenna Radiation pattern measuring results (solid line: measured data, dot line: ideal data) 


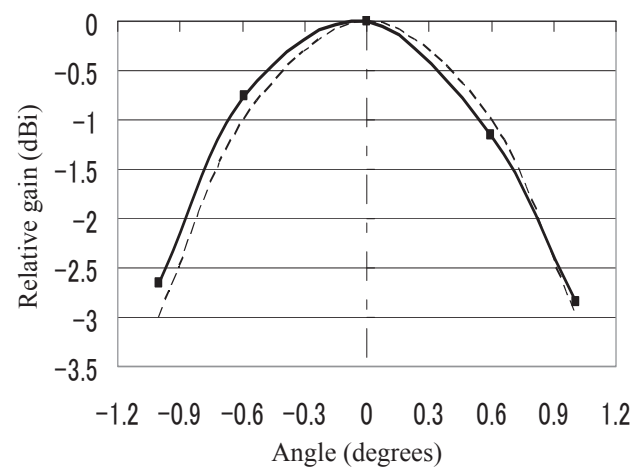

(a) East-west direction (Beam \#1)

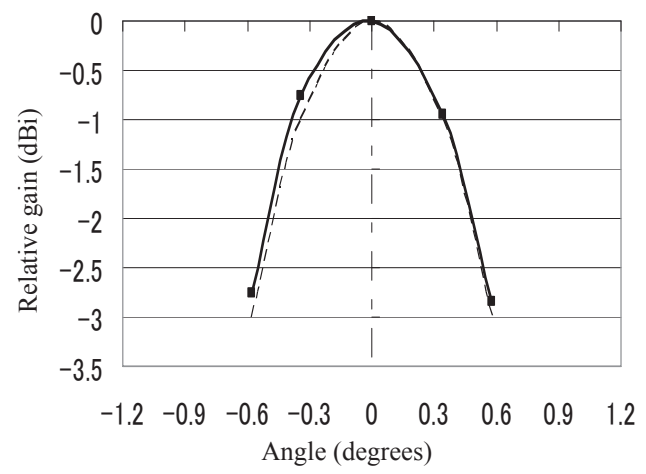

(b) North-south direction (Beam \#1)

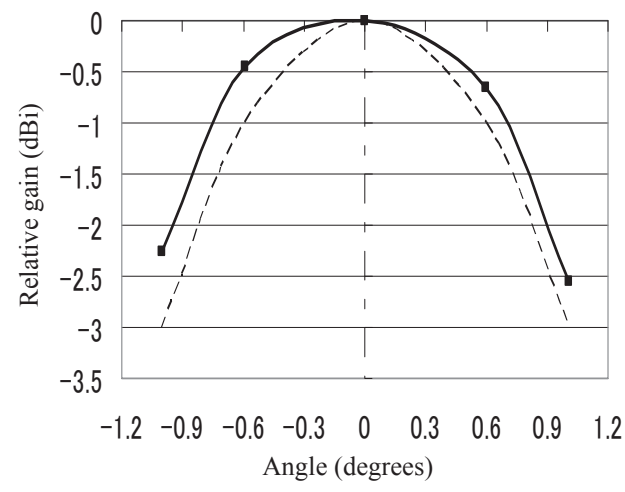

(c) East-west direction (Beam \#2)

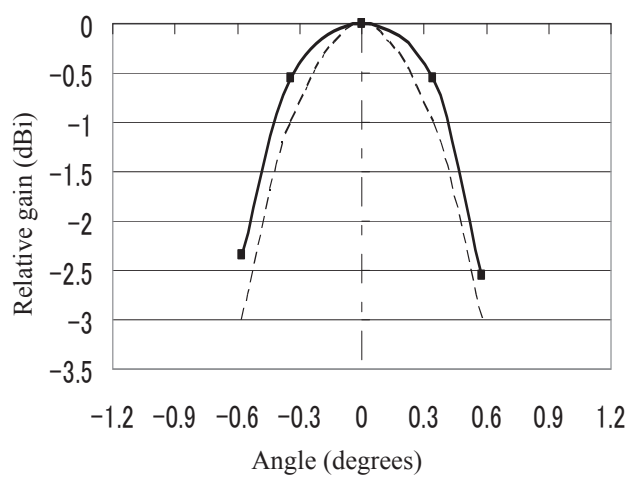

(d) North-south direction (Beam \#2)

Fig. 7. RX Antenna Radiation pattern measuring results (solid line: measured data, dot line: ideal data).
11.25 degrees. Figure 8 shows a schematic of level variation of the RF signal received at the station by the REV method. The phase of each element is changed by 11.25 degrees, from 0 to 360 degrees.

After the phase changes of one element are completed, the phase-changing process of the next element starts, and this continues for all 128 elements. There is a transient phase setting on each element operation which begins to show a distinction in one element's operation as regards the monitored RF signal level.

Figure 9 shows an example of the test results. Based on these results, and so on, all elements of the TX/RX array antenna could be considered normal. We have already performed four health check confirmation experiments (in May, October, December 2008, and March 2009) and all elements were found to be normal. The difference between measured data and expected data in the simulation is assumed to be due to satellite attitude errors and PS phase errors caused by temperature variations.

\section{Conclusion}

We have confirmed that WINDS APAA worked well by performing some communication experiments. The EIRP, G/T values are higher than the specification, and smaller earth stations can communicate using the APAA. It is a first hopping spot beam antenna in JAXA.

\section{References}

1) Yajima, M., Kuroda, T., Maeda, T. and Shimada, M.: Active Phased Array Antenna for WINDS Satellite, Proc. 25th AIAA ICSSC, AIAA-2007-3240, Korea, April 2007.

2) Maeda, T., Nakamura, Y. and Araki, T.: WINDS Satellite Bus Subsystem, Proc. 25th AIAA ICSSC, AIAA-2007-3268, Korea, April 2007.

3) Mano, S. and Katagi, T.: A Method for Measuring Amplitude and Phase of Each Radiating Element of a Phased Array Antenna, Trans. IEICE, J65-B No. 5 (1982), pp.555-560. 


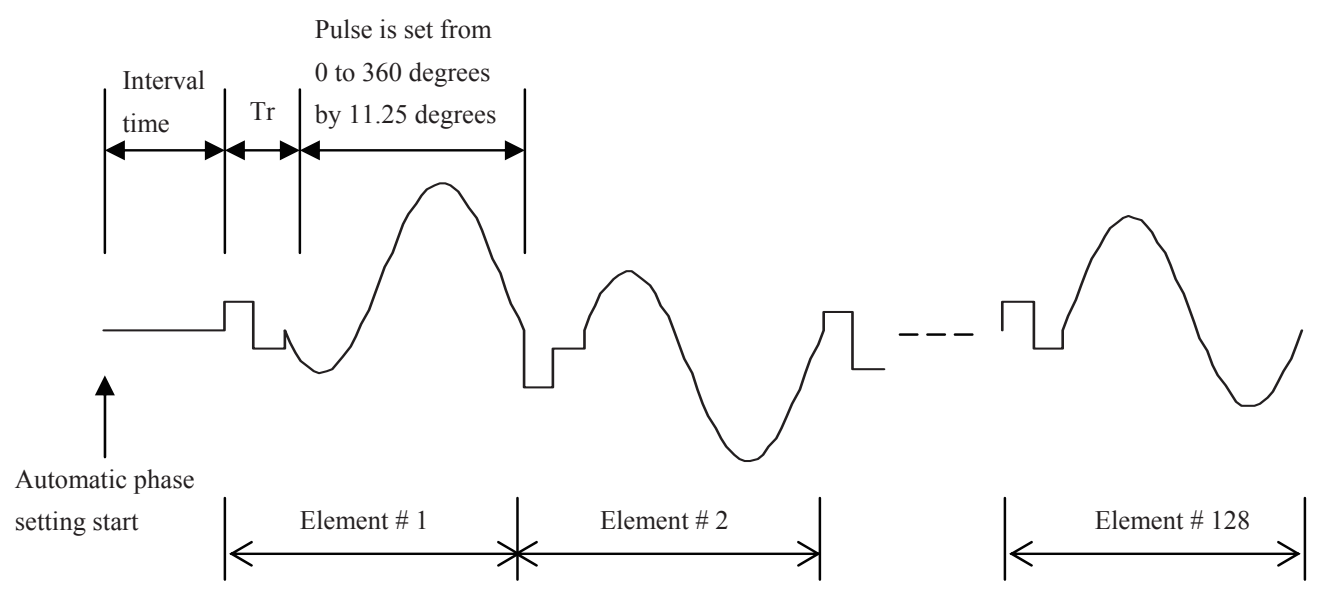

Tr: Transient phase setting ( 2 phase values)

Fig. 8. Received RF signal level variation schematic by REV method.
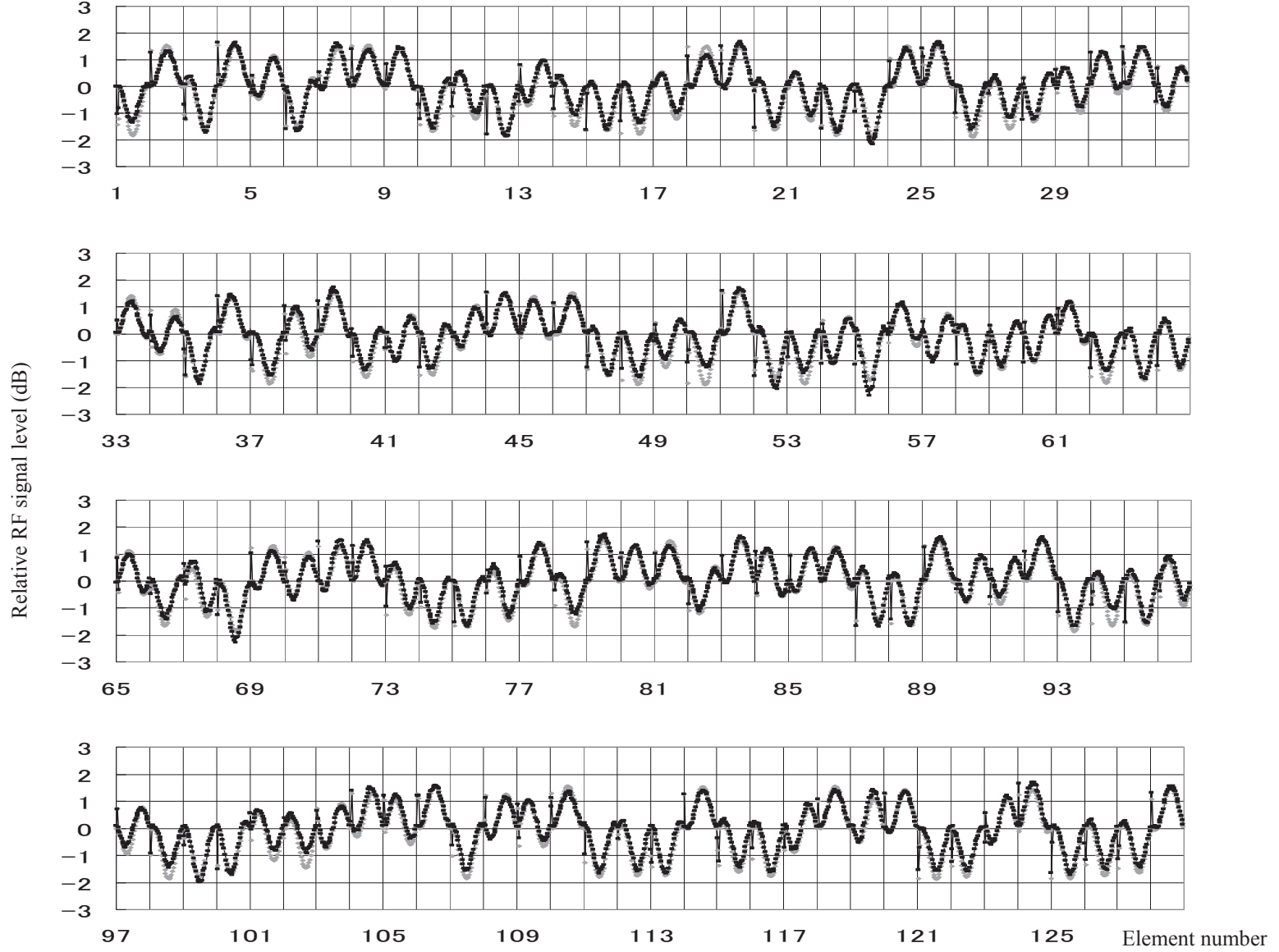

Fig. 9. Health check test results (ash line: measured data, black line: expected data in the simulation). 